\title{
Verdad y pensamiento crítico en la educación para la paz
}

\author{
Manuel Alejandro Amado*
}

\section{Introducción}

De acuerdo con la Alianza Educación para la Construcción de Culturas de Paz (2016, p. 5), la educación para la paz se entiende como un ejercicio de transformación cultural. Este ejercicio involucra prácticas de reconciliación y reintegración social, en un marco de verdad y justicia transicional; es decir, la educación para la paz es parte de los mecanismos con los que cuenta una sociedad para buscar la verdad, difundirla y resolver los problemas derivados del pasado, de actos violentos y abusos sistemáticos y, de este modo, servir a la justicia y a la reconciliación. El ejercicio de transformación social que propone la Alianza Educación contiene una serie de presupuestos teóricos que son aún materia de discusión, acerca de lo que significa la educación, la paz y lo que se requeriría para mantener o promover esta última. El presente escrito pretende ahondar en varios de estos presupuestos teóricos.

Específicamente, los objetivos de este trabajo son dos. El primero consiste en hacer una revisión de los diferentes acercamientos teóricos en torno al concepto de educación para la paz, con el propósito de defender una noción mínima que permita capturar supuestos comunes en los distintos acercamientos.

* Doctor en Filosofía de la Universidad Nacional de Colombia. Profesor de la Universidad Central (Bogotá). 
El segundo objetivo es defender una tesis acerca de cómo el concepto de búsqueda y difusión de la verdad se relaciona con el tipo de transformación que, comúnmente se asume, involucra la educación para la paz. En la literatura sobre la educación para la paz se suele asumir que la verdad es requisito para llevar a cabo esta transformación, en cuanto que tiene una función reparadora, pues permite lidiar con el resentimiento y el dolor que afectan a las víctimas y a la sociedad y, por otro lado, el conocimiento de las lecciones aprendidas del pasado evita o frena la repetición de actos violentos. Se expondrá, sin embargo, que la tesis de la función reparadora de la verdad enfrenta una serie de problemas metodológicos (varios de ellos expuestos ampliamente en la literatura sobre los procesos de posconflicto y justicia transicional ${ }^{1}$ ) que obligan a una reformulación modesta.

Para realizar esta reformulación modesta se debe contemplar una serie de distinciones y criterios que pueden ser usados para justificar razonablemente el papel reparador de la verdad. Una de estas distinciones, sugerida por Michael Ignatieff (1999, p. 164), es aquella entre verdad factual y verdad moral: por un lado, la verdad de las narraciones acerca de los hechos acontecidos y, por otro lado, la verdad de los juicios explicativos de estos hechos, es decir, la verdad de las explicaciones acerca de por qué y a causa de qué se produjeron estos hechos. Se examinará si ambos tipos de verdad, factual y moral, son indispensables para la función reparadora de la verdad y cómo la enseñanza del pensamiento crítico $^{2}$ es central en una propuesta de educación para la paz, entendida en un sentido de paz minimalista e individual, que se fundamente en la función reparadora de la verdad factual.

1 Véase, por ejemplo, ICTJ (2009), Assistance (2003), Bassiouni (2007), Beristain (2005), Byman (2002), Centro de Memoria Histórica (2012), Elster (2006), Mendeloff (2004), Roht-Arriaza y Popkin (1995), Payne (2009) y Van Zyl (1999).

2 Sobre el concepto de pensamiento crítico, véase AFA (1990), Ennis (1991), Drake (1976), Facione (2007), Feely (1976), Glaser (1941), McPeck (1981), Baron y Sternberg (1987), Brookfield (1987) y Paul (1990). 


\section{El concepto de educación para la paz}

Tanto el concepto de educación como el de paz han recibido análisis diversos. No obstante, los variados acercamientos en torno a la educación para la paz pueden agruparse en, al menos, tres tendencias conceptuales. Veremos en qué consisten estas tendencias para luego discutir las nociones de paz que involucran.

\section{Acercamientos basados en contenidos}

En términos de la clasificación de Fountain (1999, p. 39), existe un concepto de educación para la paz que involucra un conjunto de saberes proposicionales o contenidos multidisciplinarios cuya posesión permite encontrar soluciones efectivas para evitar la guerra. Los diferentes acercamientos que asumen este concepto de educación para la paz basada en contenidos difieren en el tipo de contenidos involucrados y en la manera cómo estos conocimientos permitirían encontrar soluciones para evitar la guerra. Así, por ejemplo, de acuerdo con el COPRED (Consortium on Peace Research, Education and Development) (1986), los conocimientos adquiridos permitirían desarrollar soluciones a la injusticia y promoverían la reconciliación, lo que, eventualmente, contribuiría a la paz. De acuerdo con otros acercamientos basados en contenidos, los contenidos involucrados son fundamentalmente de memoria histórica de los crímenes y abusos a los derechos humanos que (aunque no necesariamente permiten encontrar soluciones a los problemas de injusticia o promueven la reconciliación) ayudan a adquirir lecciones del pasado para evitar la repetición de actos violentos que lleven a la guerra (Hayner, 1994, p. 607). Otros acercamientos incluyen, además de lo anterior, la enseñanza de diferentes teorías acerca de las causas y soluciones a los conflictos (Regan, 1993).

\section{Acercamientos basados en habilidades}

Otro tipo de aproximaciones al concepto de educación para la paz la definen en términos, no de contenidos o saberes proposicionales teóricos, sino en términos de habilidades, destrezas, actitudes o capacidades 
no necesariamente discursivas ni proposicionales. Las habilidades o actitudes que se consideran involucradas son diversas, pero incluyen, entre otras, la tolerancia, la simpatía, la resiliencia, la capacidad de cooperar, la habilidad de resolver conflictos, la habilidad de negociar y tomar decisiones en situaciones de incertidumbre (Fountain, 1999; Cremin 1993; Chetkow-Yanoov, 1993).

\section{Acercamientos mixtos}

Finalmente, otros tipos de concepciones sobre la educación para la paz asumen una postura mixta, en el sentido de que tanto cierto conjunto de contenidos como cierto conjunto de habilidades aparecen de manera esencial en la estructura de cualquier práctica educativa enfocada en la construcción de la paz. Esta es justamente la concepción que subyace en la propuesta de la Alianza Educación para la Construcción de Culturas de Paz (2016), pues enfatiza tanto en contenidos de memoria histórica como en el fomento de habilidades, destrezas y actitudes vinculadas con la resolución de conflictos:

El fomento de la memoria y conocimiento de la historia es de vital importancia para la educación que se orienta a la construcción de culturas de paz ya que se alimenta del conocimiento y la adecuada enseñanza de las historias locales. El ejercicio de la memoria es importante para las comunidades en lo que se refiere a su pasado, pero se proyecta más ampliamente hacia el futuro como medio eficaz de lucha contra la impunidad y contra la repetición de los hechos violentos padecidos, y por otras, que de no preverlo, podrían pasar por experiencias similares. La educación para la paz se concibe como un ejercicio de transformación cultural. La polarización ideológica, el manejo violento de los conflictos y la destrucción de la naturaleza hacen necesarias prácticas de reconciliación entre las personas, las comunidades y los grupos sociales, pero también reconciliación de estos con el medio ambiente. (2016, p. 8) 


\section{Concepción mínima de la paz}

Independientemente del énfasis en los contenidos o las habilidades, las variadas posturas sobre el concepto de educación para la paz asumen presupuestos demasiado rígidos acerca de lo que implica obtener y mantener la paz, lo que en gran medida dificulta no solo extraer un núcleo común a estas propuestas, sino definir las políticas y programas que puedan aplicarlas. En lo que sigue, exhibiré algunos de estos presupuestos para extraer una concepción mínima de la paz que permita evaluar algunos de los aspectos metodológicos y epistemológicos de los tres tipos de acercamientos a la educación para la paz anteriormente expuestos.

Siguiendo el diagnóstico de Mendeloff (2004, p. 363), en la literatura sobre la construcción de paz se suelen asumir conceptos positivos o expansivos de paz. De acuerdo con estos sentidos expansivos, la paz implica la ausencia de "violencia estructural": desigualdad socioeconómica endémica, desigualdad cultural (religiosa, étnica, racial), desunión o carencia de identidad social. Asumir un concepto positivo de la paz implica aceptar una serie de compromisos acerca de lo que es necesario para construirla y mantenerla: ausencia de discriminación, democracia liberal, armonía de grupo, reconciliación, verdad, justicia (retributiva o restaurativa).

Hay al menos dos dificultades al asumir una noción expansiva de la paz y, por tanto, de asumir los robustos compromisos que implica. La primera dificultad es que no es obvio que la conexión entre los conceptos de paz, justicia, democracia y reconciliación sea una conexión analítica y, por tanto, necesaria; más bien parece una conexión meramente contingente. El segundo problema es que es incluso cuestionable que exista una relación empírica, causal, entre estos conceptos. A este respecto Byman (2002, p. 5) señala que si bien la ausencia de pobreza, discriminación, armonía, justicia y cooperación son aspectos deseables en una sociedad, la reunión de ellos no necesariamente implica ni es implicada por la paz.

Un ejemplo de estas dos dificultades lo ofrecen las observaciones del posconflicto en Sudáfrica: la CRV (Comisión de Reconciliación y Verdad) fue instaurada en Sudáfrica para esclarecer los acontecimientos 
del Apartheid. Luego de terminado el conflicto y de que las labores de la CRV fueron concluidas, la probabilidad de que se reanudara una guerra civil o un conflicto violento a gran escala era muy baja, lo que en principio sugiere un ambiente de paz; no obstante, difícilmente puede afirmarse que, luego de ese periodo, se viviera un ambiente en el que se observara la presencia de valores como la reconciliación, la armonía, la cooperación o la unidad social. Tal vez la observación de estos valores pueda servir en algunos casos de indicador de la presencia de la paz, pero no hay una clara conexión conceptual ni causal entre ellos (Byman, 2002, p. 5).

Para evitar las anteriores dos dificultades, se empleará un concepto minimalista o negativo de paz. Mendeloff (2004) sugiere definir la paz negativamente como "la ausencia de violencia o guerra organizada a gran escala y la extremadamente baja probabilidad de reanudación de la guerra" (p. 366). Esta concepción negativa incorpora dos condiciones necesarias para la paz, una de ausencia de conflicto y la otra de estabilidad. Una de las ventajas de este concepto negativo de la paz es que provee criterios mínimos para determinar si se ha logrado o no establecer la paz: si en época de posconflicto persiste un alto riesgo de reanudación del conflicto, entonces, en el sentido minimalista expuesto, no se ha logrado establecer la paz.

Siguiendo el concepto minimalista, la función de la educación para la paz, entendida en cualquiera de las tres formas (basada en conocimientos, basada en habilidades o mixta), es, como mínimo, proveer las herramientas para evitar la violencia o guerra organizada a gran escala y reducir significativamente la probabilidad de que esta se reanude. Antes de discutir cómo la educación para la paz podría contribuir al cumplimiento de estas dos condiciones, debe ser anotada otra ambigüedad en el concepto de paz.

Así como existe una distinción entre el sentido positivo o extenso y el sentido minimalista o negativo de la paz, existe también un sentido micro, o individual, y un sentido macro o global de la paz. Salomon y Nevo (1999) plantean la distinción de esta forma: por un lado, existe la ausencia de conflicto o tensión violenta al nivel del individuo y, por otro lado, existe la ausencia de conflicto o violencia al nivel de lo colectivo. Si esta distinción se aplica al concepto mínimo de la paz que se 
asumirá aquí, se debe distinguir entonces entre la ausencia de conflicto violento y baja probabilidad de su reanudación al nivel del individuo, por un lado, y al nivel global o comunal, por el otro. Idealmente, la educación para la paz nos debería proporcionar herramientas para lograr la paz a nivel micro y la paz a nivel macro, pero queda por determinar cómo puede lograr ambas y si existe una conexión entre ellas: si la paz micro contribuye a la paz macro o viceversa. En la siguiente sección se discutirán los presupuestos teóricos acerca de cómo lograr la paz, tanto global como individual, y el papel que en estas propuestas desempeñan la búsqueda y difusión de la verdad.

\section{La verdad y sus efectos para la construcción de paz}

Mendeloff (2004) realiza una exhaustiva clasificación de los presupuestos teóricos que vinculan la búsqueda y difusión de la verdad con la construcción de la paz. Estas propuestas se pueden clasificar en al menos siete categorías no excluyentes, definidas de acuerdo con los presuntos efectos que comúnmente se atribuyen a la búsqueda y difusión de la verdad:

1. Terapia y reconciliación

2. Justicia

3. Recopilación histórica oficial

4. Educación pública

5. Reforma institucional

6. Democracia

7. Denuncia y prevención

De acuerdo con las teorías de la terapia y reconciliación, la verdad ocupa un lugar central en la construcción de la paz, dado que contar la verdad sobre los crímenes cometidos durante un conflicto, o las motivaciones de estos crímenes, permite que los individuos y la comunidad lleven a cabo procesos de sanación, les ayuda a cerrar un duelo o 
a sobrellevar un trauma. Esta función terapéutica de la verdad logrará, según las teorías en cuestión, que las víctimas y victimarios empiecen a construir relaciones de reconciliación de manera adecuada. La reconciliación entre individuos implicará una reconciliación en la comunidad, lo que a su vez abonará el terreno para el fomento de la paz micro y, consecuentemente, para la paz macro.

Según las teorías de la justicia, la búsqueda y difusión de la verdad tienen el objetivo de promover justicia, restaurativa o retributiva, para las víctimas de crímenes de guerra. Al traer justicia se trae paz, ya que, presuntamente, se cierran círculos de impunidad y se disminuyen los motivos para que los individuos se vean impulsados a tomar la justicia por sus propias manos, o llevar a cabo actos violentos de venganza.

Para las teorías de la recopilación histórica, indagar por la verdad es el primer paso para la construcción de bases de información oficiales sobre crímenes o violaciones de los derechos humanos. En cuanto que son bases de datos oficiales se espera que reciban el reconocimiento del Estado y que sean objetivas. El presunto efecto de la creación de bases de información oficiales sería la reducción significativa del número de mentiras en el discurso público, lo que a su vez impediría, en primer lugar, que los medios y los demagogos inciten a la violencia a través de la manipulación de la información y, en segundo lugar, que aquellos vinculados en la promoción de actos violentos y violaciones a los derechos humanos ganen la aceptación de la comunidad (Huyse 2003, p. 30). De este modo, al menos uno de los factores que incitan a la guerra será debilitado, y parte de las condiciones para acceder a la paz será satisfecha.

Las teorías de la educación pública mantienen que difundir la historia en un Estado a través de sus instituciones permite que los individuos puedan comprender no solamente los eventos que caracterizaron un conflicto, sino las lecciones o enseñanzas que dichos eventos pueden dejar para prevenir la repetición de la violencia en el futuro (Sarkin, 1999, p. 800).

Por su parte, las teorías de la reforma institucional se comprometen con la idea de que la búsqueda de la verdad, ya sea a través de juicios o comisiones de verdad, puede revelar errores o falencias en la estructura 
institucional que facilitan los conflictos violentos o la guerra. Una vez revelados los defectos institucionales se puede diseñar reformas para corregirlos y, así, evitar la reanudación de un conflicto violento que proceda de causas institucionales.

La teoría del papel democrático de la verdad es probablemente una de las teorías más aceptadas en la literatura sobre la construcción de paz. Varias versiones de esta teoría mantienen que la búsqueda y difusión de la verdad permiten fortalecer las instituciones democráticas, ya sea porque crean un consenso sobre los eventos históricos; o porque, debido a la promoción de la justicia, fortalecen las bases legales, centrales en cualquier sistema democrático; o porque generan deliberación crítica, que es fundamental para el sustento de un régimen democrático. El supuesto aquí es que el fortalecimiento de la democracia es crucial para la construcción de la paz. Un ejemplo notable de la teoría democrática (defendida tanto globalmente como en el contexto colombiano) lo ofrece Payne (2009). Con respecto a las confesiones de los paramilitares desmovilizados, esta autora sostiene en su análisis sobre los procesos de justicia transicional en Colombia:

En lugar de considerar las confesiones como amenazas a la democracia, las presenta como catalizadores para la práctica democrática. Amplios sectores de la sociedad empiezan a participar en un diálogo sobre el pasado y su significado para la vida política contemporánea. La participación en el proceso político, la libertad para expresar opiniones contenciosas y el debate público de opiniones opuestas sobre el pasado permiten que se ponga en práctica la democracia. $(2009$, p. 312)

Las teorías de la denuncia y prevención mantienen que la condena, e incluso el solo señalamiento público de criminales de guerra, tiene un efecto preventivo en cuanto que aísla a los criminales de la sociedad, los somete al escarnio o vergüenza públicos, envía un mensaje de alerta a la sociedad y de disuasión para potenciales criminales. Toda vez que la condena y la disuasión actúen como dispositivos de prevención de conflicto, estos dispositivos servirán a los propósitos de la paz. 
En la literatura sobre la construcción de paz es posible encontrar acercamientos complejos que combinan dos o más de las teorías expuestas sobre los efectos de la verdad sobre la paz. No obstante, como señala Mendeloff (2004), la mayoría de estos acercamientos no tienen suficiente evidencia empírica, ni bases argumentativas, para sustentar sus afirmaciones causales sobre los efectos de la verdad en la construcción de paz. Por las mismas razones, cualquier propuesta de educación para la paz que asuma alguna o varias de las teorías sobre los efectos de la verdad, enfrentará problemas metodológicos o epistemológicos, pues no contará con la evidencia empírica o justificación argumentativa para sustentar el papel de la verdad en la transformación cultural que pretende.

Es particularmente notable, por ejemplo, que varias propuestas de educación para la paz, como la propuesta de Unicef (Fountain, 1999), y la expuesta ampliamente por la Alianza Educación para la Construcción de Culturas de Paz (2016), asuman el presupuesto teórico de la terapia y reconciliación. Como se señaló más arriba, este presupuesto mantiene que la búsqueda y difusión de la verdad cumplen un papel en la construcción de la paz, dado que permiten que los individuos y la sociedad realicen un proceso de cierre que cura heridas psicológicas y, de este modo, abona el terreno para facilitar procesos de reconciliación, procesos que causen o que sean requeridos para la construcción de paz.

La evidencia empírica, no obstante, parece desmentir o al menos poner en duda el supuesto teórico del papel terapéutico y reconciliatorio de la verdad. En primer lugar, la difusión de la verdad no logra, en números casos de confesiones de crímenes de guerra, abonar un terreno para la reconciliación. El mismo estudio de Payne (2009), que analiza las confesiones recogidas por comisiones de la verdad en cinco países (Argentina, Brasil, Chile y Sudáfrica), muestra que:

La esperanza que se expresa en la literatura de la justicia transnacional — que las confesiones de los perpetradores deben conducir hacia la reconciliación, definida como la resolución de conflictos del pasado o como un acuerdo amistoso entre bandas rivales- pasa 
por alto las diferencias a menudo irreconciliables entre las víctimas y los perpetradores. Más que pedir disculpas por sus actos, los perpetradores tienden a racionalizarlos y a minimizar su responsabilidad personal, aumentando así la tensión sobre el pasado, en vez de disminuirla. $(2009$, p. 2$)$

En segundo lugar, la difusión de la verdad no parece tener, en muchos casos, un valor terapéutico que contribuya al cierre de heridas psicológicas o traumas, y más bien puede resultar contraproducente al ser un factor de revictimización; es decir, promueve nuevas violaciones a los derechos de las víctimas e implica la repetición de experiencias traumáticas. En el caso de conflictos en los que la difusión de la verdad ocupó un papel preponderante en el posconflicto, como en el caso del Apartheid, no existe evidencia empírica que demuestre que las víctimas hayan mostrado mejoría psicológica a largo plazo luego de escuchar los testimonios recopilados por la comisión de reconciliación; contrario a esto, los datos anecdóticos sugieren que pueden sufrir un trauma inmediato y duradero (Allan y Allan, 2000, p. 472). Por otro lado, los testimonios de los victimarios no suelen limitarse a la confesión de crímenes de guerra, sino a la racionalización de los mismos de una forma en la que se privilegia la perspectiva del perpetrador, elevándolo, en algunos casos, a la categoría de héroe y justiciero sádico, como ha ocurrido en numerosos testimonios de victimarios en Argentina, Brasil, Chile y Colombia (Payne, 2009). Además, los testimonios de victimarios no siempre revelan la verdad, ya sea porque ocultan información o porque crean ficciones para defender a sus cómplices o a ellos mismos (Comisión Intereclesial de Justicia y Paz, 2010; Payne, 2009).

Es generalmente aceptado que las víctimas, así como la sociedad, tienen el derecho a conocer los eventos ocurridos en el pasado. No obstante, el hecho de que la justificación o racionalización de los crímenes cometidos por los victimarios sea una fuente probable de revictimización, parece entrar en tensión con el presunto valor terapéutico de la verdad. La conclusión, sin embargo, no debe ser negativa en el sentido de que la búsqueda y difusión de la verdad no tiene, por sí misma, un valor terapéutico, pues la verdad que tiende a revictimizar no es necesariamente la verdad de los hechos acontecidos. Para ver por 
qué, puede ser útil acudir a la distinción que Michael Ignatieff (1999, p. 164) traza entre verdad factual y verdad moral: por un lado, está la verdad de las narraciones acerca de los hechos acontecidos y, por otro lado, la verdad de los juicios explicativos de estos hechos; es decir, la verdad de las explicaciones acerca de por qué y a causa de qué se produjeron estos hechos. Así, aunque las declaraciones o testimonios en los que se supone se revela la verdad moral tienden a producir revictimización y a perder, así, su valor terapéutico, no necesariamente sucede lo mismo en las declaraciones que revelan exclusivamente la verdad factual, pues en estas se suprimen las racionalizaciones y justificaciones de los crímenes cometidos, con lo que se evitan las declaraciones de heroísmo o sadismo. Sugiero entonces que la búsqueda y difusión de la verdad aún podría tener un valor terapéutico y, por tanto, un valor para la paz en el sentido mínimo y micro (paz como ausencia de conflicto y baja probabilidad de reanudación a nivel individual, por oposición al nivel global o comunitario). Este resultado se aplica a cualquier acercamiento de educación para la paz: en principio, la educación para la paz podría gestar procesos de construcción de paz en un sentido mínimo e individual a través de la búsqueda y difusión de la verdad factual.

Pero ¿̇de qué modo la educación para la paz (incluso en el sentido mínimo e individual), puede construir paz mediante la búsqueda y difusión de la verdad (factual)? Incluso bajo la limitación del concepto minimalista e individual de la paz, y la restricción de la búsqueda y difusión de la verdad en su sentido factual, la educación puede tener un papel central en la construcción de la paz. Como lo señala Mendeloff (2004, p. 356), existe evidencia empírica que señala "la creación de mitos" (mythmaking), es decir la construcción sistemática de mentiras (disfrazadas muchas veces con el manto del chauvinismo o del hipernacionalismo) y distorsiones deliberadas de los hechos históricos, o verdades factuales, como coadyuvante en la generación de guerras entre Estados y en el interior de ellos. De modo que cualquier iniciativa que reduzca o prevenga la creación de mitos puede ayudar a prevenir la guerra o la reanudación de la misma, y consecuentemente, ayudar a la construcción de la paz en un sentido minimalista e individual. En la siguiente sección se sugerirá cómo la enseñanza del pensamiento 
crítico puede ocupar un lugar central en la construcción de la paz, en el sentido enfatizado, al brindar herramientas que permiten detectar y evitar la creación de mitos.

\section{Pensamiento crítico, verdad factual y paz minimalista individual}

Del mismo modo que hay una variedad de acercamientos al concepto de educación para la paz y la construcción de paz, hay una buena cantidad de acercamientos a lo que significa el pensamiento crítico y cómo puede ser promovido. A pesar de la multitud de teorías, es ampliamente admitido que el pensamiento crítico involucra la toma de decisiones y la formulación de juicios como resultado del ejercicio de un conjunto de "habilidades cognitivas y disposiciones conductuales" (AFA, 1990).

Entre las habilidades cognitivas involucradas en el ejercicio del pensamiento crítico se encuentran: "la interpretación, análisis, evaluación, inferencia, explicación y autoregulación” (Facione, 2007, p. 4). No hay criterios de individuación que delimiten precisamente cada una de estas habilidades, pero pueden identificarse algunos elementos centrales que las caracterizan. La interpretación no se limita a la decodificación de significados, es también la habilidad de comprender y expresar la relevancia de una idea o experiencia en un contexto determinado, la capacidad de aclarar el sentido de afirmaciones y cursos de acción, lo que implica, a su vez, la habilidad de parafrasear las ideas de otros y determinar sus intenciones comunicativas. El análisis incluye la capacidad de detectar argumentos, esto es, tener la habilidad de detectar una tesis y las razones a favor o en contra de la misma, así como la habilidad de encontrar las ideas centrales en un texto o discurso. También incluye la capacidad de comparar puntos de vista para determinar sus similitudes y diferencias. La evaluación, por su parte, es la capacidad de determinar la validez y juzgar la credibilidad de argumentos, razones, evidencias, testimonios y presuntas autoridades. La inferencia, como su nombre lo indica, es la capacidad de encontrar las relaciones inferenciales entre diferentes contenidos, lo que supone la capacidad de formular hipótesis, identificar 
compromisos epistémicos tanto de las creencias de otros como de las propias, detectar la coherencia o compatibilidad entre enunciados, y trazar consecuencias deductivamente válidas o inductivamente confiables. La explicación involucra la capacidad de describir resultados, creencias y cursos de acción, de tal forma que se justifiquen los procedimientos, la elección de métodos, fuentes y conexiones causales elaboradas. Finalmente, la autorregulación es una capacidad metacognitiva, es decir, es una habilidad que se aplica a otros procesos cognitivos o a los resultados de estos. De esta forma, la autorregulación involucra el examen de las propias ideas (argumentos, decisiones, cursos de acción, procedimientos), la capacidad de identificar y cuestionar los propios prejuicios, y evitar la influencia de sesgos cognitivos, tanto egocéntricos como sociocéntricos.

Las habilidades cognitivas señaladas son solamente parte de la ecuación de lo que implica el pensamiento crítico. Es posible que una persona tenga estas habilidades, pero que no las aplique en absoluto, no las aplique en los contextos apropiados (como en situaciones de riesgo o peligro inminente en el que se requiere una acción rápi$\mathrm{da}$, instintiva), o que las aplique en contextos demasiado restringidos (por ejemplo, solamente en el aula de clase). También es posible que estas habilidades se debiliten por la falta de práctica sostenida o por condiciones psicofísicas. En consecuencia, para el ejercicio efectivo del pensamiento crítico no es suficiente la posesión de las habilidades cognitivas mencionadas, se necesita la exhibición de una serie de disposiciones conductuales. Entre estas disposiciones se cuenta la de ser inquisitivo (disposición a buscar la verdad), sistemático, de mente abierta (reconocer la existencia de opiniones contrarias y modificar la propia ante la presencia de argumentos o evidencias razonablemente fuertes), confiar en el razonamiento y tener disposición a la discusión argumentada (Facione, 2007).

A partir de la presente caracterización del pensamiento crítico se puede ver cómo el ejercicio de las habilidades y disposiciones que lo componen es central para construir la paz en, al menos, el sentido minimalista individual. Un individuo que ejercite las habilidades de pensamiento crítico es capaz de identificar creencias débilmente sustentadas, prejuicios culturales o teóricos sin fundamento empírico 
o racional, como lo son buena parte de las creencias resultado de la creación de mitos. Estas creencias están fundadas en ideales fundamentalistas sin justificación racional, extremo nacionalismo o $\mathrm{ce}$ guera teórica (incapacidad de apreciar las falencias o excepciones de una teoría). Un pensador crítico es también capaz de modificar sus creencias y revisar sus prejuicios ante evidencia contundente, capacidad que no es exhibida por quienes mantienen acríticamente ideas difundidas por un líder de opinión o por medios de comunicación masiva. Un pensador crítico difícilmente motivaría sus decisiones, o las decisiones de los demás, apelando a métodos represivos o intimidatorios. Adicionalmente, dado su interés en buscar la verdad, su habilidad para interpretar, analizar y evaluar, un pensador crítico no podrá ser fácilmente convencido de presuntas verdades históricas o factuales que no estén soportadas por la argumentación y las fuentes apropiadas.

Por consiguiente, la posesión de habilidades y disposiciones de pensamiento crítico puede evitar y reducir notablemente la creación de mitos, o la influencia que esta ejerce sobre un individuo. Y dado que, como se señaló al final de la sección anterior, evitar o reducir la construcción de mitos promueve la paz (al menos en el sentido minimalista e individual), se sigue que la posesión de las habilidades y disposiciones propias del pensamiento crítico promueve la paz. Una educación para la paz que cultive el pensamiento crítico será, por tanto, una educación que permita la construcción de la paz.

\section{Educación para la paz y transferencia del pensamiento crítico}

Hay dos preocupaciones relacionadas con la enseñanza del pensamiento crítico que afectan cualquier acercamiento de educación para la paz que busque promoverlo. En primer lugar, se encuentra la pregunta acerca de si las habilidades y disposiciones del pensamiento crítico pueden ser desarrolladas o promovidas. En segundo lugar, existe la preocupación de cómo lograr que estas habilidades se transfieran a múltiples contextos, es decir, que el desarrollo de estas habilidades 
vaya acompañado por el ejercicio de las mismas en contextos sociales mucho más amplios y diversos que un aula de clase o un determinado dominio del saber.

Con respecto a la primera preocupación, es posible encontrar una serie de estudios que recogen evidencia para mostrar que, efectivamente, las habilidades y disposiciones propias del pensamiento crítico pueden ser promovidas y mejoradas (Baron y Sternberg, 1987; Brookfield, 1987; Chetkow-Yanoov, 1996; Perkins y Salomon, 1989).

Con respecto a la segunda preocupación, existe un debate álgido sobre qué tan dependientes del contexto pueden ser las habilidades del pensamiento crítico y la real posibilidad de su transferencia a múltiples contextos. Sin embargo, existen desarrollos teóricos positivos. Uno de los acercamientos positivos más notables al problema de la transferencia de las habilidades del pensamiento crítico es realizado por Halpern $(1998,2001)$. Esta autora toma como base teórica varios de los modelos que la psicología cognitiva emplea para explicar el aprendizaje, la memoria y el procesamiento de información en humanos.

A partir de esta base teórica, Halpern propone un modelo de enseñanza de habilidades propias del pensamiento crítico con miras a la transferencia. Esquemáticamente, el modelo consta de cuatro partes:

1) Un componente disposicional en el que se discriminan y enseñan los elementos necesarios para decir cuándo es adecuado, y cuándo no, aplicar habilidades de pensamiento crítico para la resolución de un problema, por ejemplo, elegir qué ropa interior ponerse en un día normal no demanda tanta capacidad de procesamiento como elegir qué tipo de vivienda adquirir o en qué invertir una considerable cantidad de dinero.

2) Instrucción y práctica de habilidades de pensamiento crítico, que consiste en la instrucción explícita sobre los tipos de habilidades de pensamiento crítico, con el propósito de caracterizarlas y permitir que se reconozcan a partir de la resolución de problemas que requieren el ejercicio de una o varias de estas habilidades.

3) Entrenamiento en el reconocimiento de la estructura de problemas de diferentes contextos. La idea aquí es que la transferencia de habilidades de pensamiento crítico se facilita al enseñar a reconocer patrones estructurales compartidos en problemas que provienen de 
diferentes contextos. Así, por ejemplo, varios problemas que requieren la toma de decisiones tienen una estructura similar. Dada esta semejanza estructural, una decisión razonada en cualquiera de los problemas evitará la falacia de los costos hundidos. Esta falacia consiste en pensar que las inversiones pasadas son relevantes para decisiones que solamente implican costos futuros: invertir millones para arreglar un auto viejo solamente porque un amigo ha invertido otros tantos no es una buena decisión; lo mismo que la decisión tomada por un congreso al invertir sumas exorbitantes en un programa militar porque el ejército ya ha invertido sumas similares, o lo mismo que decidir casarse con alguien solamente porque ya se han pasado muchos años juntos. La estructura de los tres problemas de decisión (arreglar un carro viejo, invertir en un programa militar, casarse) es la misma, aunque los problemas sean distintos. En los tres, sin embargo, dada su estructura, una decisión razonada evitará caer en la falacia de los costos hundidos. Diseñar estrategias que permitan reconocer la estructura de problemas en diversos dominios o contextos facilitará, de acuerdo con Halpern (1998, p. 453), la transferencia de las habilidades cognitivas requeridas para resolverlos.

4) La última parte del modelo de enseñanza de Halpern es un componente metacognitivo, cuyo propósito es el de coordinar y evaluar el propio pensamiento. Como se expuso más arriba, el pensamiento crítico requiere el uso de habilidades de autorregulación que permitan evaluar y dirigir los propios procesos de pensamiento y toma de decisiones. El modelo en consideración propone que se promueva la autoconciencia a través de preguntas que requieran la descripción explícita de procesos de pensamiento que usualmente ocurren de forma implícita o desatendida. Por ejemplo, dado un problema, preguntar por el tipo de habilidades que se cree son necesarias para resolverlo, el tiempo aproximado que podría tardar en resolverse, el progreso relativo que se tiene mientras se está buscando una solución, o en qué momento y cómo se llegó a averiguarla.

El modelo de enseñanza de habilidades de pensamiento crítico de Halpern no se aplica a un único dominio del saber, por lo cual puede ser usado para dar cuenta de cómo un acercamiento a la educación para la paz que incorpore la promoción de habilidades de pensamiento 
crítico puede, en principio, ofrecer herramientas para lograr la transferencia de esas habilidades a contextos sociales más amplios que el aula de clase.

\section{Conclusiones}

Las consideraciones hechas hasta aquí acerca de cómo una educación para la paz que cultive el pensamiento crítico puede motivar la paz se aplican, como fue señalado anteriormente, a una noción minimalista y micro de la paz, entendida como la ausencia de conflicto y baja probabilidad de reanudación del mismo a nivel individual. Se esperaría que una contribución a la paz en sentido individual tenga repercusiones sobre la paz en un sentido global, comunitario. Sin embargo, es arriesgado pensar que existe una conexión inferencial obvia entre la paz individual y la paz global, y se carece de investigaciones empíricas que liguen causalmente un concepto al otro, por lo que los resultados presentados en este artículo son limitados en ese respecto.

Adicionalmente, debe considerarse que, a pesar de que la búsqueda y difusión de la verdad son fundamentales en un acercamiento a la educación para la paz como el aquí esbozado, el tipo de verdad en el que se hace énfasis es la verdad en su sentido factual. Aunque no se desestima que las habilidades propias del pensamiento crítico permitan reducir las consecuencias indeseables o revictimizantes de la verdad moral (como las que ocurren en los casos de testimonios de crímenes de guerra), el presente texto no se compromete con que el pensamiento crítico permita reducir los efectos psicológicos adversos que muchas veces acompañan la búsqueda y difusión de esta verdad.

A pesar de lo anterior, si la argumentación expuesta hasta aquí es sólida, un acercamiento a la educación para la paz que privilegie la verdad factual y pretenda prevenir o disminuir la eficacia de la creación de mitos, es un acercamiento que no solo debe estar basado en conocimientos, también en habilidades y, por tanto, según la clasificación hecha en la segunda sección, debe ser un acercamiento mixto. El tipo de habilidades promovido en una educación para la paz en el sentido aquí expuesto debe incluir, aunque no necesariamente de forma exclusiva, 
las habilidades y disposiciones que caracterizan el pensamiento crítico. Dichas habilidades son enseñables y, en principio, transferibles a una diversidad de contextos amplios.

\section{Referencias bibliográficas}

Alianza Educación para la Construcción de Culturas de Paz (2016). Orientaciones acerca de la inclusión participativa de la educación para la construcción de culturas de paz en los planes de desarrollo territoriales. Recuperado de http://www.educacionparalapaz.org.co/recursos2. htm? $\mathrm{x}=18938004$

Allan, A. y Allan, M. (2000) 'The South African Truth and Reconciliation Commission as a Therapeutic Tool'. Behavioral Sciences and the Law $18,459-477$.

Asociación Filosófica Americana [AFA] (1990). Pensamiento crítico: una declaración de consenso de expertos con fines de evaluación e instrucción educativa.

Baron, J. B. y Sternberg, R. J. (1987). Teaching thinking skills: Theory and practice. New York: W. H. Freeman.

Bassiouni, M. C. (2007). Los Principios de Chicago sobre Justicia Transicional. Recuperado de http://www.law.depaul.edu/centers_institutes/ihrli/ pdf/chicago_principles.pdf

Beristain, C. M. (2005). Reconciliación luego de conflictos violentos: un marco teórico. Verdad, justicia y reparación: desafíos para la democracia y la convivencia social (pp. 15-52). San José: Instituto Internacional para la Democracia y la Asistencia Electoral; Instituto Interamericano de Derechos Humanos.

Bloomfield, D., Barnes, T. y Huysem, L. (eds.) (2003). Reconciliation After Violent Conflict A. Handbook. (pp. 10-173). Estocolmo: International Institute for Democracy and Electoral Assistance.

Brookfield, S. (1987). Formando Pensadores Críticos: Retando a Los Adultos a Que Exploren Modos Alternos de Pensar y de Actuar. San Francisco, CA: Josey-Bass Publishers.

Byman, D. (2002). Keeping the Peace: Lasting Solutions to Ethnic Conflicts. Baltimore: Johns Hopkins University Press. 
Centro de Memoria Histórica (2012). Justicia y Paz: ¿ Verdad judicial o Verdad histórica? Bogotá: Centro de Memoria Histórica.

Chetkow-Yanoov, B. (1996). Conflict-Resolution Skills Can be taught. Peace Education in a Postmodern World, 71(3), 12-28.

Comisión Intereclesial de Justicia y Paz (2010). Los efectos psicosociales de la revictimización. Recuperado de http://justiciaypazcolombia.com/ Los-efectos-psicosociales-de-la

Consortium on Peace Research, Education and Development [COPRED] (1986). Report on the Juniata Process. COPRED Peace Chronicle.

Cremin, P. (1993). Promoting education for peace. En P. Cremin (ed.), Education for Peace. Educational Studies Association of Ireland and the Irish Peace Institute.

Drake, J. A. (1976). Teaching critical thinking: Analyzing, learning and teaching critical thinking skills. Danville: Interstate.

Elster, J. (2006). Rendición de cuentas: La justicia transicional en perspectiva histórica. Buenos Aires: Katz.

Ennis, R. H. (1991). Critical thinking: A streamlined conception. Teaching Philosophy, 14, 5-25.

Facione, P. (2007). Pensamiento Crítico: ¿Qué es y por qué es importante? California: Insight Assessment California Academic Press.

Feely, T. (1976). Critical thinking: Toward a definition, paradigm, and research agenda. Theory and Research in Social Education, 4, 1-19.

Fountain, S. (1999). Peace Education in UNICEF. Nueva York: Unicef.

Glaser, E. M. (1941). An experiment in the development of critical thinking. New York: Teachers College, Columbia University.

Halpern, D. F. (1998). Teaching critical thinking for transfer across domains: Dispositions, skills, structure training, and metacognitive monitoring. American Psychologist, 53(4), 449-455.

Halpern, D. F. (2001). Assessing the effectiveness of critical thinking instruction. The Journal of General Education, 50(4), 270-286.

Hayner, P. (1994). Fifteen Truth Commissions, 1974 to 1994: A Comparative Perspective. Human Rights Quarterly, 16, 597-655.

Huyse, L. (2003). The Process of Reconciliation. En D. Bloomfield, T. Barnes y L. Huyse (eds.) Reconciliation after Violent Conflict: A Handbook. Estocolmo: International Institute for Democracy and Electoral Assistance. 
Ignatieff, M. (1999). El honor del guerrero: Guerra étnica y conciencia moderna. Buenos Aires: Taurus.

International Center for Transitional Justice (2009). ¿Qué es la Justicia Transicional? Recuperado de http://ictj.org/es/que-es-la-justicia-transicional

Mcpeck, J. (1981). Critical thinking and education. Nueva York: St. Martin's Press.

Mendeloff, D. (2004). Truth-Seeking, Truth-Telling, and Postconflict Peacebuilding: Curb the Enthusiasm? International Studies Review, 6, 355-380.

Paul, R. (1990). Critical thinking: What every person needs to survive in a rapidly changing world. Rhonert Park, CA: Center for Critical Thinking and Moral Critique.

Payne, L. (2009). Testimonios perturbadores: Ni verdad ni reconciliación en las confesiones de violencia de Estado. Bogotá: Universidad de los Andes.

Perkins, D. N. y Salomon, G. (1989). Are cognitive skills context-bound? Educational Researcher, 18, 16-25.

Regan, C. (1993). Peace education: a global imperative. En P. Cremin (ed.), Education for Peace. Limerick: Irish Peace Institute.

Roht-Arriaza, N. y Popkin, M. (1995). Truth as Justice Investigatory Commissions in Latin America, 20 Law \& Social Inquiry, 79.

Salomon, G. y Nevo, B. (1999, noviembre). Peace Education: An Active Field in Need for Research. Peace Education Conference, Haifa, Israel.

Sarkin, J. (1999). The Necessity and Challenges of Establishing a Truth and Reconciliation Commission in Rwanda. Human Rights Quarter$l y, 21,767-823$.

van Zyl, P. (1999). Dilemmas of Transitional Justice: The Case of South Africa's Truth and Reconciliation Commission. Journal of International Affairs, 52(2), 21. 
